\title{
Recovering current density from data on electric potential
}

\author{
J. Irša, A. N. Galybin \& A. Peratta \\ Wessex Institute of Technology, Southampton, UK
}

\begin{abstract}
In this paper, an inverse problem for galvanic corrosion in two-dimensional Laplace's equation was studied. The considered problem deals with experimental measurements on electric potential, where due to lack of data, numerical integration is impossible. The problem is reduced to the determination of unknown complex coefficients of approximating functions, which are related to the known potential and unknown current density. By employing continuity of those functions along subdomain interfaces and using condition equations for known data leads to over-determined system of linear algebraic equations which are subjected to experimental errors. Reconstruction of current density is unique. The reconstruction contains one free additive parameter which does not affect current density. The method is useful in situations where limited data on electric potential are provided.
\end{abstract}

Keywords: current density, potential measurements, reconstruction, complex variables.

\section{Introduction}

The evaluation of field of current density is essential in problems of galvanic corrosion. In many cases the direct measurement of current density is not feasible, while the electric potential can be obtained from experimental measurements. This is particularly true in case of cathodic protection systems in general, where many surveying techniques (for example DCVG and CIS for underground structures) rely in potential measurements at different points at the electrolyte in order to identify the current distribution along the metallic structures. 
In these cases, it is common that the region of interest for collecting data is not accessible to the operator and in order to exploit the available measurements some indirect method is required.

There are many studies that imply numerical methods for the forward modelling of galvanic corrosion problem. These techniques are based mainly on boundary value problems (BVP) formulations in order to obtain or verify results, such as finite element method (FEM), finite difference method (FDM) or boundary element method (BEM). These methods are successfully used and showed to be very accurate to solve BVPs. Some of them are also implemented in commercial software.

Situations may arise, where the boundary conditions are unknown and only some experimental data in certain locations are known. In this case, the problem is defined as an inverse one. This situation often occurs in many branches of science and mathematics where only the values of some model parameters can be obtained from observed data or measured data. Data on electric potential can be obtained in galvanic corrosion as a set of discrete data with one free parameter due to measuring potential differences. This situation, where measurements on electric potential can be provided as a set of discrete data within simply connected domain $\Omega$ imposes the problem to be inverse.

Theory of complex variables $[1,2]$ is used in order to connect current density and electric potential with one holomorphic function. The real part of it represents electric potential and the derivative is related with current density. The domain is divided into smaller subdomains where the holomorphic function is approximated with quadratic function and its derivative with piece wise linear function. These approximating functions obey continuity across subdomain interfaces.

Similar method was used in [3] with heat flow and in [4, 5] with stress trajectories. The purpose of this work is to develop efficient method for identification of current densities within a domain where some data on electric potential are provided.

\section{Galvanic current density distribution in electrolyte}

Current density is a measure of the density of flow of a conserved charge. The equation governing the distribution of potential and current flow in electrolyte can be derived from the continuity equation, charge conservation. The divergence of the current density is equal to the negative rate of change of the charge density [6]:

$$
\nabla \cdot I=-\frac{\partial q}{\partial t}
$$

For steady state current distribution, $\partial q / \partial t=0$ in uniform isotropic electrolyte, where the conductivity of electrolyte is constant, the electric potential satisfies Laplace's equation: 


$$
\Delta \phi=0
$$

The current density can be defined at any point as:

$$
I_{j}=-k \frac{\partial \phi}{\partial x_{j}}
$$

The fact that electric potential $\phi(x, y)$ is harmonic, which comes from (2) allows to of use theory of complex variables, for 2D potential problems. Introducing harmonic conjugate function $\psi(x, y)$, where both of them satisfy CauchyRiemann equations (CR), one has holomorphic function:

$$
W(z)=\phi(x, y)+i \psi(x, y)
$$

where the real part is electric potential and the imaginary part is function harmonic conjugated, related with fluxes. $\phi(x, y)=$ const. are equipotentials and $\psi(x, y)=$ const. flux lines. Using CR and transformation rules in calculus, one can see that the derivative of holomorphic function $W$ is related to the current density as follows:

$$
H(z) \equiv \frac{\partial W}{\partial z}=\frac{\partial \phi}{\partial x}-i \frac{\partial \phi}{\partial y}
$$

Comparison of (3) and (5) shows that derivative of holomorphic function, which has real part equal to electric potential is related to the conjugated current density $\widetilde{I}$ in the form $H(z)=\widetilde{I} /-k$.

\section{Problem description}

The problem is to recover the current density, based on experimental data on electric potential. Let us consider a domain $\Omega$, to be a simply connected domain or a subdomain of a bigger domain which is not necessarily simply connected and bounded. Let potential measurements be known at some discrete points $(j=1, \ldots, N)$, located within $\Omega$. The general problem is formulated as follows: given the discrete data on electric potential $\phi_{j}=1 \ldots N$ find field of current density within the domain $\Omega$. There are no other restrictions, such as data type or boundary conditions. The data can be redistributed uniformly or randomly.

\subsection{On uniqueness of reconstructed solution}

The problem deals with reconstruction of a holomorphic function, where the real and imaginary parts are harmonic and satisfy CR. Therefore having one function known, the second one can be easily obtained by integration of CR. Indeed, assume, for instance, that the real part of the holomorphic function is known analytically $\phi(x, y)$. CR allows finding the former by integration: 


$$
\psi(x, y)=\int \frac{\partial \phi(x, y)}{\partial x} d y+p
$$

This formula indicates that the reconstruction can be achieved but it will always have one undetermined parameter as a free parameter. In this particular case is the free parameter an additive constant in the imaginary part $\psi(x, y)$ of holomorphic function $W(z)$. After performing derivative of $W(z)$, the free parameter vanishes. It is therefore obvious that from electric potential one can recover the current density without any free parameter. In the case when data are known at discrete points, reconstruction, in general, is non-unique and can have any finite number of free parameters, which is evident from the following. Let an exact solution $f(z)=u(x, y)+i v(x, y)$ of the problem be found by any method. It is obvious, that the modulus must be real; therefore it satisfies the following:

$$
\operatorname{Im}\left[f\left(z_{j}\right) e^{-i \omega\left(z_{j}\right)}\right]=0, j=1 \ldots N
$$

where $\omega$ is argument of $f(z)$. Let us consider a function $P(z)$ in the form:

$$
P(z)=\prod_{j=1}^{m} C_{j}\left(z-z_{j}\right)^{m_{j}}
$$

where $C_{j}$ are arbitrary complex parameters, $m_{j}$ are arbitrary positive integers and $z_{j}$ are the data points. It is evident from (8) that the sum, $f(z)+P(z)$, satisfies the condition (7) and therefore it is also the solution of the problem.

In order to have sense the solution has to be sought in certain class of functions. Hereafter the solution is sought as a piecewise linear holomorphic functions. It will be shown that this set of functions allows reconstructing the current density with high accuracy.

\section{Mathematical model}

Similar mathematical formulation has been described in [3], where the required function was related to the modulus of holomorphic function. In this case is the required current density related directly with the derivative of holomorphic function $W(z)$.

The domain $\Omega$ is divided into $n$ smaller subdomains (elements) of an arbitrary shape, as shown in Figure 1. In order to achieve piecewise linear function of current density, the function $W(z)$ must be approximated by quadratic function. Both these approximations obey continuity along the interfaces (at certain collocation points located on the interfaces). 


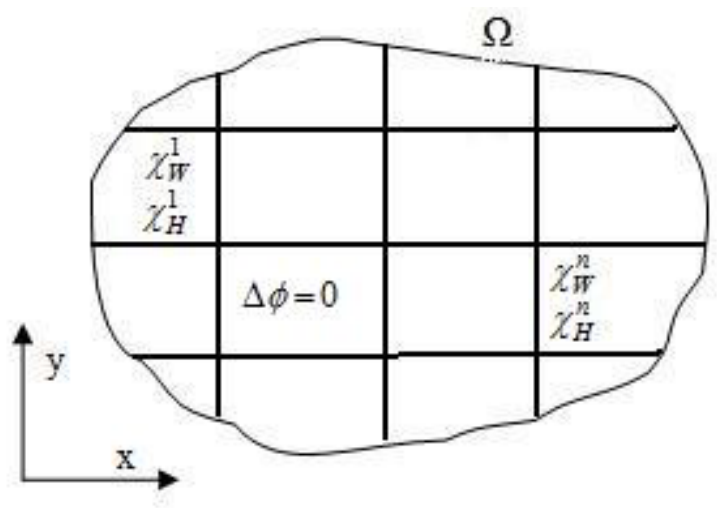

Figure 1: Discretization of the domain with approximating functions.

For function $H(z)$, related to current density is approximating function required to be piece-wise linear function:

$$
\chi_{H}^{(m)}(z)=a^{(m)}+b^{(m)} z, \quad m=1 \ldots n
$$

for function $W(z)$, which has real part electric potential is therefore the approximating function the integration of $H(z)$ :

$$
\chi_{W}^{(m)}(z)=c^{(m)}+a^{(m)} z+\frac{1}{2} b^{(m)} z^{2}, \quad m=1 \ldots n
$$

Here $a^{(m)}=a_{0}^{(m)}+i a_{1}^{(m)}, b^{(m)}=b_{0}^{(m)}+i b_{1}^{(m)}, c^{(m)}=c_{0}^{(m)}+i c_{1}^{(m)}$ are 3 unknown complex constants; therefore 6 real constants are unknown in every element. The method is not restricted to these two particular approximating functions, however it will be demonstrated that the use of these two particular functions provides results with very high accuracy.

\subsection{Discretization of the domain}

In order to obey the continuity of the above mentioned functions, a grid was used consisting of square elements with collocation points (CP) on its interfaces. The $\mathrm{CP}$ are distributed regularly.

The minimum number of nodes strictly depends on the number of unknowns in the approximating functions as well as the element type and number of known data. Should the system consist only from the continuity equations and no data imposed on electric potential, one can find the number of required collocation points as follows. Given that, the square elements are placed in the regular grid with $J$ elements along $x$-axis and $K$ elements along $y$-axis and 6 unknown real coefficients in every element, one finds the total number of unknowns $N_{\text {unkn }}$ as:

$$
N_{\text {unkn }}=6 N_{\text {elem }}=6 \mathrm{JK}
$$


The required number of collocation points depends on the number of element interfaces, which is $N_{\text {int }}=(J-1) K+(K-1) J$. Using the same number of collocation points per each interface, keeping in mind that separation of complex equations into real and imaginary parts presents two real equations at every collocation point, the required number of collocation points per interface is:

$$
N_{c p} \geq \frac{N_{\text {unkn }}}{2_{\text {int }}}=\frac{6 J K}{2[(J-1) K+(K-1) J]}=\frac{3 J K}{2 J K-J-K}
$$

For the domain, where $J$ or $K$ is equal to 1 , the number of collocation points per interface is $N_{c p} \geq 3$. In general case the number of collocation points per interface $N_{c p}>1$, therefore $N_{c p}=2$ is used further on. Given dense data on electric potential, one could reduce the number of $\mathrm{CP}$ with respect to unknowns and total number of equations.

Other types of mesh could be used, such as triangular or polygonal with the straight or curvilinear sides. The square grid is convenient from computational point of view because the distances between collocation points are regular, which has good influence on the structure of the matrix of the system.

\subsection{Equations of continuity and condition equations}

The problem consists of continuity equations and two types of condition equations. For the $k$-th collocation point, lying on the interface between the elements numbered $m$ and $m+1$, the equation of continuity for $W(z)$ is following:

$$
\chi_{W}^{(m)}\left(z_{k}\right)-\chi_{W}^{(m+l)}\left(z_{k}\right)=0, \quad m=1 \ldots n ; \quad k=1 \ldots N_{C P}
$$

The second equation of continuity for $H(z)$ is expressed following:

$$
\chi_{H}^{(m)}\left(z_{k}\right)-\chi_{H}^{(m+l)}\left(z_{k}\right)=0, \quad m=1 \ldots n ; \quad k=1 \ldots N_{C P}
$$

The condition equation containing data on electric potential $\phi$ comes from $W(z)$, where the real part of it is equal to the electric potential, therefore:

$$
\operatorname{Re}\left(\chi_{W}^{(m)}\left(z_{j}\right)\right)=\phi_{j}, \quad j=1 \ldots N
$$

this condition equation is satisfied in elements, where the data occurs. It is obvious that from the collocation method, one can find the differences between interfering elements coefficients only. In the condition equation (15) are presented all coefficients except the imaginary part of $c$, it stays undetermined and from the continuity equations can not be obtained uniquely. This coefficient is the free additive parameter discussed in section 3.1. This would decrease the rank of matrix and would require regularisation procedures. In order to avoid these procedures which contain additional parameters as regularization parameters etc., second condition equation is used. The second condition 
equation is due to free parameter $c_{1}$, it provides the average of all constants $c_{1}$ across whole domain zero. The condition is following:

$$
\sum_{i=1}^{N} \operatorname{Im}\left(c^{(i)}\right)=0
$$

The system of linear algebraic equations (SLAE) is formed by equations (13)(16).

\subsection{Solution of the linear system}

By extracting the real and imaginary parts of all equations (equations of continuity, condition equations) the real SLAE can be obtained and rewritten in a matrix form:

$$
A x=b
$$

where $A \in R^{m x n}, b \in R^{m}$ and $m>n . x$ is the vector of the unknown real coefficients, of length $n$. The vector $x$ consists of real and imaginary parts of unknown complex coefficients. Vector $b$ is known exactly, while, $A$ the matrix of the SLAE depends on CP, type and size of element. The matrix $A$ is not a square, the system is over-determined and therefore the left-hand side $A x$ does not exactly equal to $b$ and thus the system is inconsistent. However an approximate solution, $x^{*}$, can be found by means of the least squares methods [5] that minimise the residual:

$$
\|A x *-b\|_{2} \leq \varepsilon
$$

where $\|. .\|_{2}$ stands for the $L_{2}$ norm. If the arising system is well-posed and not large, then the inversion of the matrix does not meet any difficulties and solution takes the form [7]:

$$
x^{*}=\left(A^{T} A\right)^{-1} A^{T} b
$$

The condition number $(C N)$ is used in the numerical examples to control wellposedness of the SLAE. In the case of high $\mathrm{CN}$, the methods for ill-posed problems should be used [8].

\section{Numerical analysis}

For the purpose of testing the method, synthetic function was chosen in the form:

$$
W(z)=54\left(z-\frac{i}{3}\right)-30\left(z-\frac{i}{3}\right)^{3}
$$

Taking the real part and derivative of (20) one has the electric potential and current density for ideal function, which are used as given data (electric potential) and for comparison with numerically obtained result (current density). 
Conductivity of electrolyte presents multiplicative parameter to the current density, which was chosen as $\mathrm{k}=1$. Different types and structure of data were tested (ideal data and data subjected to errors).

\subsection{Ideal data}

This example uses data obtained from (20). It was used 81 data uniformly redistributed within the domain and 25 elements (Figure 2).

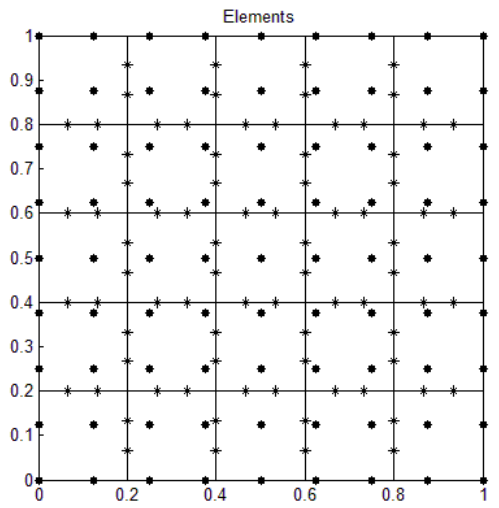

Figure 2: Discretized domain with given data on electric potential.
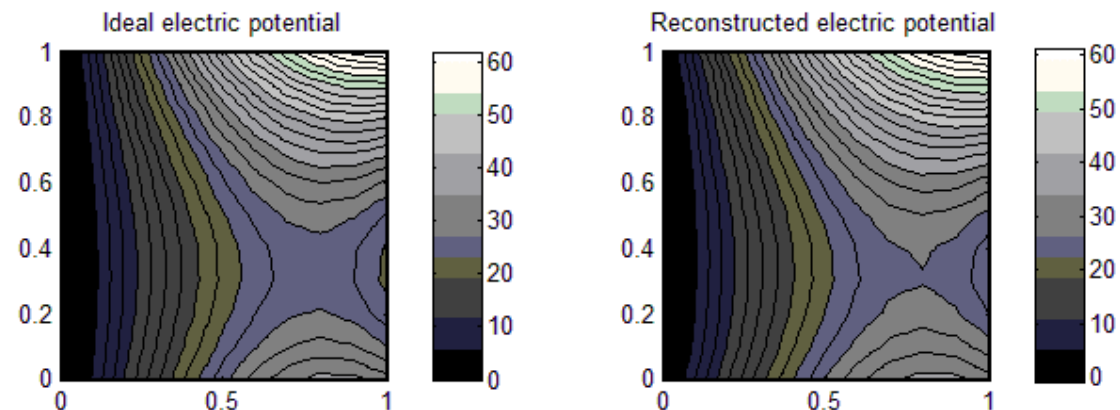

Figure 3: Ideal electric potential - left and reconstructed electric potential right.

Reconstructed electric potential and current is shown in Figures 3 and 4.

This configuration revealed accuracy with average error $4.7 \%$ by using 25 elements only. From Figure 5 can be seen the improvement with refinement in residual, maximum and average errors as well as behaviour of condition number $(\mathrm{CN})$. 

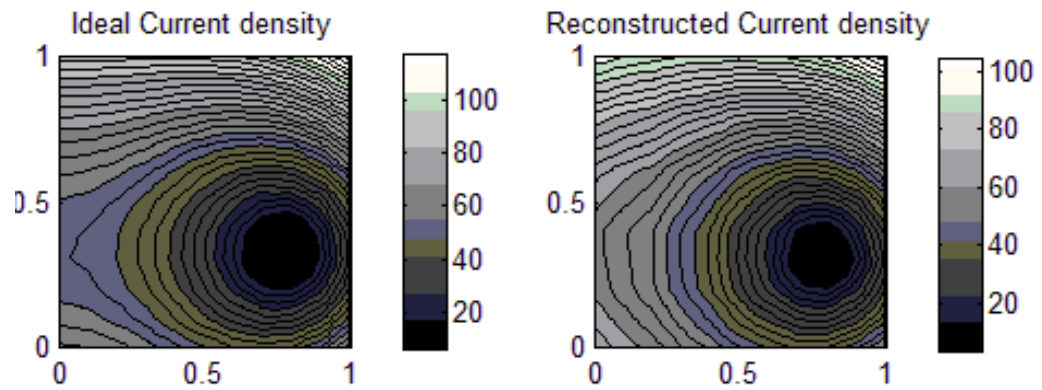

Figure 4: Ideal current density - left and recovered current density - right.

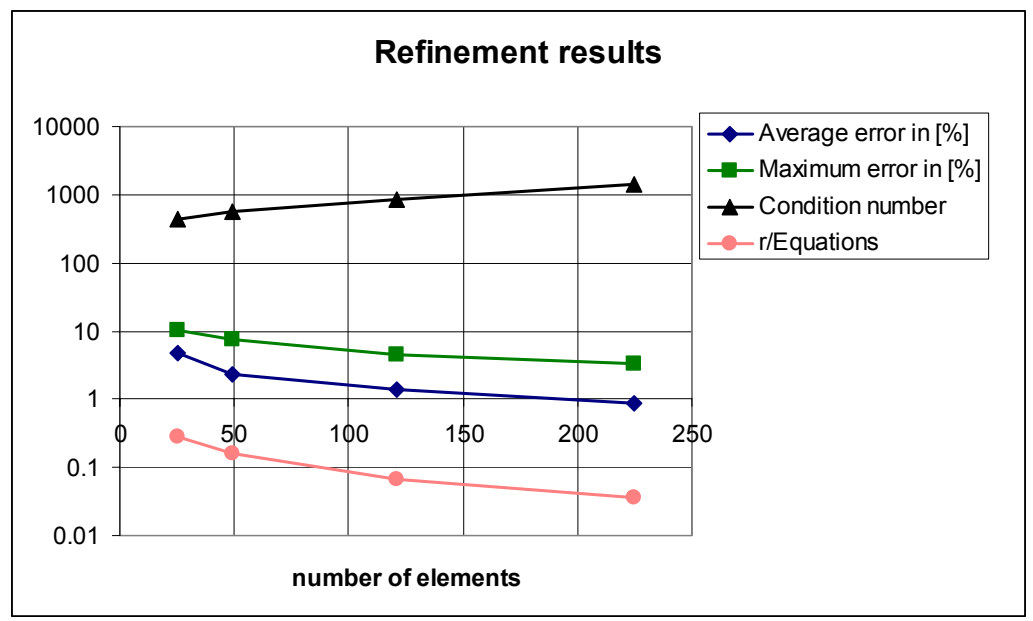

Figure 5: Refinement results for 81 ideal data.
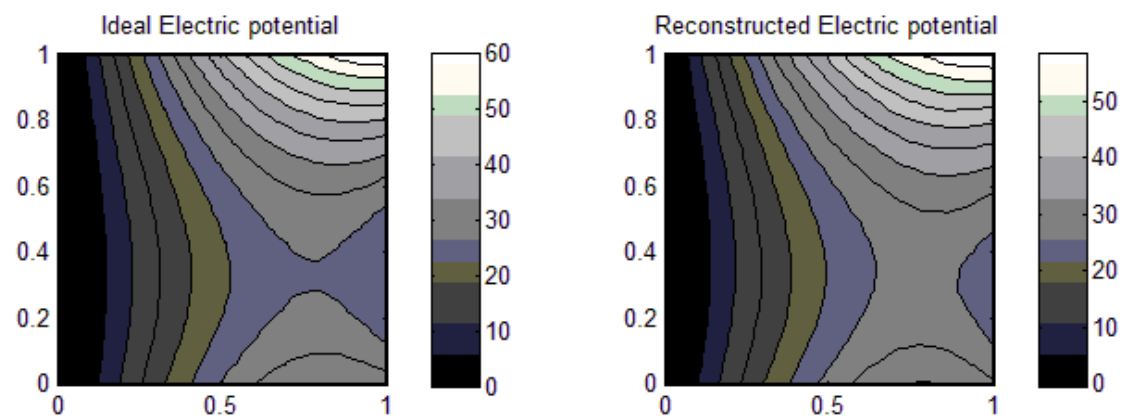

Figure 6: Ideal electric potential - left and reconstructed electric potential from data subjected to errors - right. 


\subsection{Data subjected to errors}

Random error was introduced to the potential data to simulate experimental errors. It was used error $\pm 10 \%$ on 81 uniformly redistributed data and 49 elements. The results for electric potential and current density are shown in Figures 6 and 7. The Average error was 2.9\%. Condition number was 571 and r/Eq. was 0.2943 .
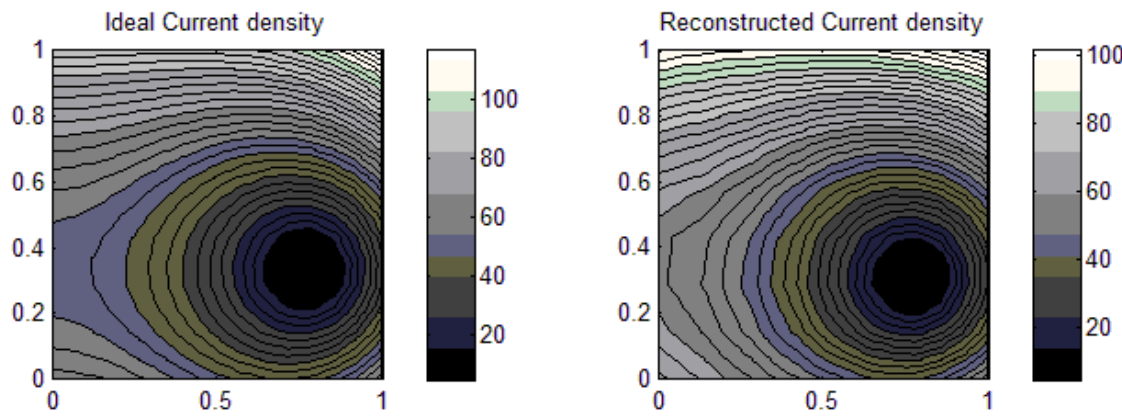

Figure 7: Ideal current density - left and recovered current density from data subjected to errors - right.

\subsection{Random data subjected to errors}

The measurements are usually randomly redistributed. Random data subjected to errors $\pm 10 \%$ were used. It was used 81 data and 49 elements (Figure 8.). The results are shown in Figure 9. Average error was 3.8\%. CN was 754 and $\mathrm{r} / \mathrm{Eq} .=0.2672$.

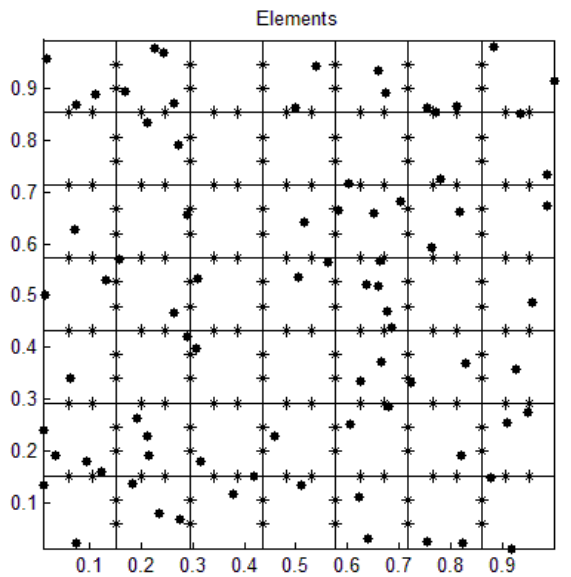

Figure 8: 81 random data subjected to errors and discretization. 


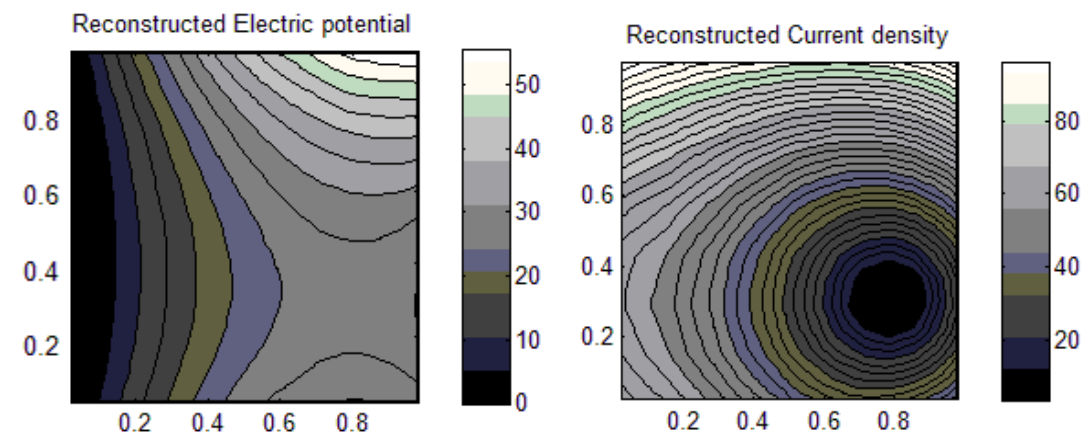

Figure 9: Reconstructed electric potential - left and recovered current density - right.

\section{Conclusion}

The recovering of current density from data on electric potential, satisfying Laplace's equation was studied. In experiments, it is difficult or expensive to obtain many measurements and therefore numerical integration cannot be performed. The recovered results revealed high accuracy with synthetic ideal function, as for ideal data, so does for data subjected to high errors. The method uses complex variable theory where one can obtain holomorphic function, related to the electric potential and its derivative related with the current density.

\section{Acknowledgement}

The authors are grateful to EPSRC for the financial support of this work through the Research Grant EP/E032494/1.

\section{References}

[1] M. Rahman, Complex Variables and Transform Calculus, Computational Mechanics Publications, Southampton, 1997.

[2] Y.K. Kwok, Applied complex variables, Cambridge University Press, Cambridge, 2002.

[3] J. Irsa, A.N. Galybin, Heat flux reconstruction in grinding process from temperature data, Computational Methods and Experimental Measurements XIV, WIT Press, Southampton, 2009.

[4] A.N. Galybin and Sh.A. Mukhamediev, Determination of elastic stresses from discrete data on stress orientations, IJSS, 41 (18-19), 2004, 5125 5142 .

[5] Sh.A. Mukhamediev, A.N. Galybin and B.H.G. Brady, Determination of stress fields in elastic lithosphere by methods based on stress orientations, INT J ROCK MECH MIN,43 (1), 2006, 66-88. 
184 Simulation of Electrochemical Processes III

[6] J.X. Jia, G. Song, A. Atrens, D.St John, J. Baynham and G. Chandler, Evaluation of the BEASY program using linear and piecewise linear approaches for the boundary conditions, Materials and Corrosion, Vol. 55, Is. $11,845-852$.

[7] G.H. Golub, C.F. Van Loan, Matrix Computations, London: The John Hopkins Press Ltd., 1996.

[8] A.N. Tikhonov, V.Y. Arsenin, Solution of Ill-posed Problems, Winston, Wiley, New York, 1977. 